that this approach shows potential as a tool for differentiating between patients with and without cancer and could even distinguish between cancers of different underlying pathologic states.

Original article Liu W et al. (2005) Using tree analysis pattern and SELDI-TOF-MS to discriminate transitional cell carcinoma of the bladder cancer from noncancer patients. Eur Urol 47: 456-462

\section{Long-term effects of pelvic nerve-sparing radical surgery}

Results of a recent study of long-term urologic and sexual function following pelvic nerve-sparing radical surgery for rectal cancer suggest that this type of surgery preserves long-term voiding function.

Ameda et al. assessed the responses of 52 male and female patients who had previously undergone the surgery and who completed a self-administered questionnaire regarding subsequent changes in their urinary-tract symptoms. Topics covered by the questionnaire included aspects of storage such as urinary frequency and urgency, and aspects of voiding such as straining. Additionally, information was collected regarding quality of life and male sexual function post-surgery.

The vast majority of patients $(92 \%)$ reported that they had been able to maintain voluntary voiding in the long term without the need for catheterization, and around $60 \%$ of patients reported no change in urinary-tract symptoms after surgery. Most patients reported that they were satisfied with their current urinary status, although this was the case for a significantly higher proportion of female patients than male. The main area of dissatisfaction postsurgery was found to be male sexual function, with $64 \%$ of males reporting that they were unsatisfied with their current sexual function.

The authors conclude that despite the successful long-term outcomes described for this type of surgery in terms of preservation of urinary-tract function, serious problems relating to postoperative erectile dysfunction still exist.

Original article Ameda K et al. (2005) The long-term voiding function and sexual function after pelvic nervesparing radical surgery for rectal cancer. Int $J$ Urol 12: 256-263

\section{Scrotal dartos flap wrapping technique reduces complications of hypospadias surgery}

Hayashi and co-workers report the application of a scrotal dartos flap wrapping surgical technique to prevent the occurrence of urethrocutaneous fistula in 14 patients with hypospadias.

Urethrocutaneous fistula is a frequent complication after surgery to repair hypospadias, particularly in severe cases where the neourethra is long. Hayashi et al. applied a scrotal dartos tunic wrap to the neourethra during one-stage urethroplasty. The dartos fascia and the tunica vaginalis containing a testis were separated using Churchill's technique for obtaining dartos tissue, but without opening the tunica vaginalis. This modified technique avoids exposure of the testis, and the abundant blood supply in the well-vascularized dartos flap improves the vascular supply to the neourethra.

Hypospadias was successfully repaired without fistula formation in all but one patient who developed a tiny fistula, and this was successfully closed by a simple procedure. No other complications occurred in the neourethra, scrotum or testis of patients who underwent surgery. Limitations included the requirement for a penoscrotal incision, unsuitability for treatment of distal urethoplasty and a longer surgery time ( $45 \mathrm{~min}$ ) compared with alternative procedures.

Hayashi et al. conclude that the scrotal dartos flap wrapping technique has fewer complications than other surgical procedures and they recommend its application for prevention of urethocutaneous fistula on hypospadias.

Original article Hayashi et al. (2005) Scrotal dartos flap for the prevention of the urethrocutaneous fistula on hypospadias urethroplasty. Int J Urol 12: 280-283

\section{Thermo-chemotherapy for bladder cancer}

The combination of local hyperthermia of the bladder wall and intravesical mitomycin-C (MMC) can be used to treat recurrent superficial transitional-cell carcinoma of the bladder effectively and safely, according to a new study by Moskovitz et al. 